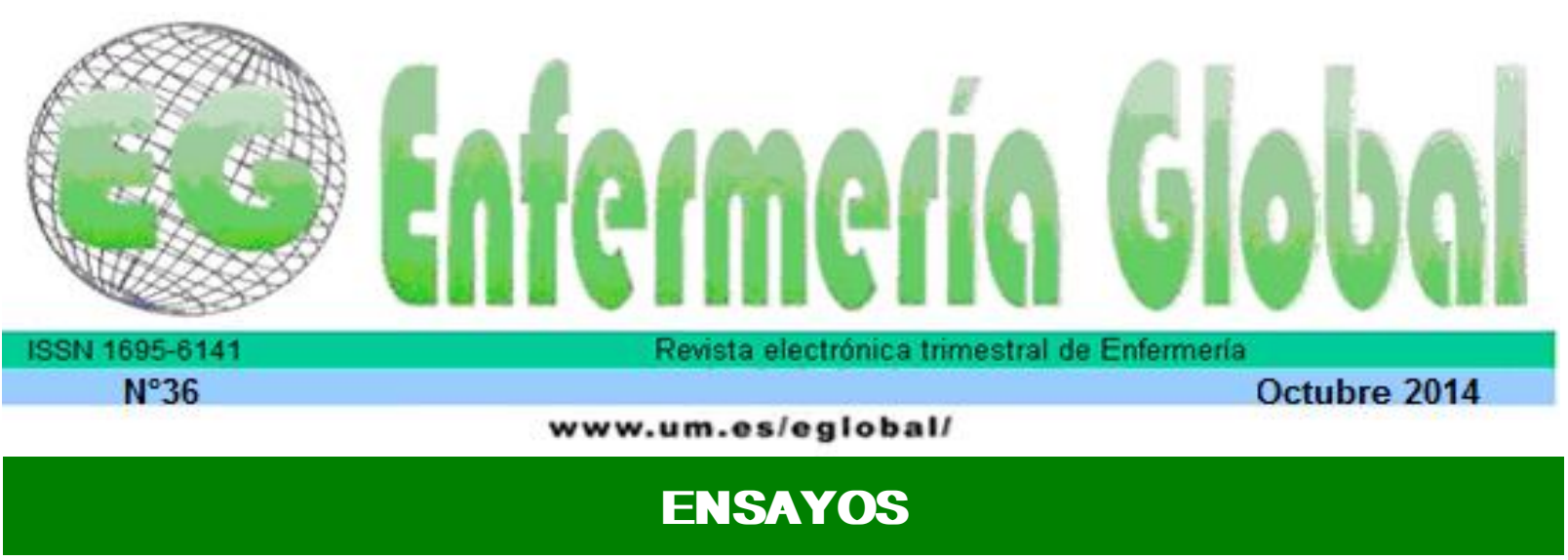

\title{
Dificultades para el avance de las mujeres. Diferentes teorías sociológicas
}

Difficulties for the advancement of women. Different sociological theories

\author{
*Montesó Curto, Pilar
}

*Facultad de Enfermería. Universidad Rovira i Virgili. Tarragona. E-mail: mariapilar.monteso@urv.cat

Palabras clave: Patriarcado; teoría estructural-funcionalista; feminismo.

Keywords: Patriarchy; structural-functionalist theory; feminism

\section{RESUMEN}

Introducción: Es importante conocer el concepto de patriarcado para entender las dificultades con que se encuentran las mujeres en el avance profesional, social e institucional. La profesión enfermera junto con otras también femeninas se ha enfrentado también con estas dificultades.

Metodología: Introduciremos varias teorías que analizan el concepto de género. Algunas teorías justifican la socialización diferencial: estructural-funcionalista (Durkheim, Marx, Engels, Weber, Parsons, Comte...) y el feminismo critica al patriarcado como elemento de dominación para las mujeres (Firestone, Myrdal, B.Ehrenreich...).

Analizaremos como las dificultades debidas a la instauración del patriarcado y a la diferente socialización entre hombres y mujeres ha frenado el avance de algunas profesiones femeninas, siendo la profesión enfermera una de ellas.

Resultados: En la familia patriarcal que ha existido en nuestra sociedad contemporánea los hombres han ocupado las posiciones más relevantes del poder político, económico y cultural quedando las mujeres en segundo plano.

Conclusión: Las desigualdades siguen existiendo en nuestras sociedades transicionales trayendo consigo un aumento de la violencia para las mujeres y con la globalización, una sociedad cada vez más dualizada con sectores más empobrecidos en los que se encuentran mayoritariamente las mujeres.

\section{ABSTRACT}

Introduction: It is important to understand the concept of patriarchy to understand the difficulties women face in career advancement, social and institutional. The nursing profession with other women's work has also been faced with these difficulties.

Methodology: We introduce several theories that analyze the concept of gender. Some justify the differential socialization theory: structural-functionalism (Durkheim, Marx, Engels, Weber, Parsons, 
Comte...) and the feminist critique of patriarchy as an element of dominance for women (Firestone, Myrdal, B. Ehrenreich...).

We will discuss the difficulties caused by the establishment of patriarchy and the different socialization of men and women has slowed the progress of some female professions, the nursing profession being one of them.

Results: In the patriarchal family that has existed in our modern society people have developed the most important positions of political power, economic and cultural women running in the background.

Conclusion: Inequalities still exist in transitional societies resulting in a rise in violence for women and globalization, a society increasingly dualized with most impoverished sectors in which majority are women.

\section{INTRODUCCIÓN}

\section{Patriarcado}

Las mujeres han desarrollado a lo largo de la historia funciones importantísimas para el mantenimiento de la humanidad y a pesar de ello no aparecen ni en los libros de texto ni en la historia sino esporádicamente. Ellas han estado devaluadas en la ciencia, tecnología, arte, literatura... y han ocupado aquellos espacios considerados privados y por tanto de poco valor. Las mujeres a la vez que adquirían conocimientos iban ocupando puestos de trabajo en los que la dualización no había desaparecido y la mayoría continuaban siendo de poca importancia social aunque la cualificación iba aumentando.

El continuado proceso de lucha y de reconocimiento hacía que las mujeres buscaran un espacio exterior en el que identificarse pero al mismo tiempo sin abandonar aquellas responsabilidades para las que habían sido educadas, como ser madres, cuidado del hogar... Este doble papel que había que mantener les creaba cierta angustia personal que habían de soportar. Para sobrevivir las mujeres debieron adoptar múltiples estrategias en una sociedad que iba cambiando pero en la que el patriarcado estaba fuertemente arraigado en su organización social. La igualdad debía ser constantemente legitimada, y defendida.

Según Castells, el patriarcado es una de las estructuras básicas de todas las sociedades contemporáneas. Se caracteriza por la autoridad, impuesta desde las instituciones, de los hombres sobre las mujeres y sus hijos, en la unidad familiar. Para Castells es el patriarcado el principal elemento en la dominación de la mujer ${ }^{1}$. Sin la familia patriarcal el patriarcado quedaría desenmascarado como dominación y acabaría siendo derrocado por la rebelión de las mujeres sometidas a lo largo de la historia.

Pero ¿cómo se mantiene esa dominación? Vivimos inmersos en una sociedad que nos va dando instrucciones algunas evidentes y otras subliminales o inconscientes, en las que podríamos incluir la teoría de Freud y Chodorow. ${ }^{2}$

Para Freud la aparición de la identidad de género se basa en el aprendizaje en los bebés sobre si tienen pene o no lo tienen. La presencia o ausencia de pene simboliza la masculinidad o la feminidad. El aprendizaje de género dice estar en torno a los cuatro o cinco años. Para Chodorow, la masculinidad se define en función de una pérdida de apego hacia la madre. La identidad masculina se configura a través de la separación, de este modo los hombres en su vida posterior y de manera inconsciente, 
sienten que su vida corre peligro si establece relaciones emocionales estrechas con los demás.

Para Giddens en los últimos años, las teorías sobre la socialización y rol de género se han visto criticadas por un número creciente de sociólogos. En vez de considerar el sexo como algo determinado biológicamente y el género como algo que se aprende culturalmente, señalan que debemos considerar ambas cosas como productos construidos socialmente. Giddens se centra en la sociedad patriarcal y cultura androcéntrica. En la sociedad patriarcal son los hombres los que ocupan las posiciones más relevantes del poder político, económico, cultural, quedando las mujeres en segundo plano.

\section{ANÁLISIS DE TEORÍAS SOCIOLÓGICAS}

\section{Teorías Sociológicas}

No sólo el género sino el sexo se ha construido socialmente, esto ha llevado a la división de roles femeninos y masculinos, siendo los masculinos recompensados y los femeninos devaluados. Esto ha sido analizado desde las diferentes teorías sociológicas que van desde la teoría estructural-funcionalista representada por Durkheim y Parsons, a la marxista contenida en los testos de Marx y Engels y Habermas, la teoría crítica de J.Habermas, la fenomenología representada por Schutz, Berger, Luckman.

El origen de la división de los géneros en los funcionalistas se encuentra en las sociedades industriales cuando hombres y mujeres realizaban actividades de caza y recolección de alimentos. Las mujeres tenían menos movilidad por causa de embarazo, parto y crianza de los hijos. Los hombres, con mayor movilidad se responsabilizaron de aportar alimentos a la familia.

Los hombres, a pesar de la importancia de las tareas agrícolas y recolectoras de las mujeres, fueron adquiriendo un papel dominante como proveedores de carne y protectores del grupo contra agresiones externas. Así se fue consolidando un modelo de relación de dependencia con una mayor valoración de todo aquello que realizaban los machos.

Para Durkheim la familia es importante para la cohesión social. Comte entiende la subordinación doméstica basada en la ley natural como modelo de todo poder social, entre las cuales no pueden encontrarse ni las mujeres ni los proletarios, dada su inferioridad ante los varones de la clase burguesa dirigente. Para Parsons la estabilidad normativa se ejercía mediante una socialización diferencial a niños y a niñas.

Para Marx y Engels lo fundamental era la posición en el proceso de producción aunque sí reconocían la discriminación de las mujeres en el proceso de producción. Weber ahonda en la comprensión de la dominación patriarcal. $Y$ afirma que ésta, basada en la sumisión de las mujeres y los hijos a la autoridad de un "dominus" dentro de una comunidad doméstica, se concreta en una devoción rigurosamente personal hacia un hombre en particular y obtiene legitimidad debido a su carácter cotidiano y a que las normas de obediencia asentadas en la tradición la hace significar como natural. K. Millet recogiendo los presupuestos de Weber formula su teoría del 
patriarcado basada en el control de la mitad de población (los hombres) sobre la otra mitad (las mujeres), aludiendo al dominio de un grupo sobre otro.

Para S. Firestone la base material que genera las relaciones de dominación es la reproducción, entendida como trabajo. Esta división natural del trabajo hizo que los hombres desarrollaran solo la mitad de sí mismos, de modo que mientras en los hombres se hipertrofia la racionalidad y la actividad, en las mujeres se hipertrofió el sentimentalismo y la pasividad.

Las mujeres han sido discriminadas al igual que otros colectivos minoritarios.

La Escuela de Chicago se convierte en impulsora de los estudios de minorías: inmigrantes, negros y finalmente las mujeres. La desaparición de un sistema paternalista para dar paso a uno regido por valores democráticos impulsado por la revolución industrial cambió el status tanto de los negros como de las mujeres. Las semejanzas históricas y estructurales entre los negros en una cultura dominada por los blancos, y entre las mujeres en una cultura masculina, fueron destacadas por Myrdal. Él les atribuye unos rasgos comunes: una inteligencia inferior, una naturaleza emocional o primitiva, una ilusoria habilidad sexual. Ambos grupos se ven inducidos a recurrir a las mismas tácticas de acomodación: una forma insinuativa de agradar a los demás, cierta tendencia a estudiar los puntos débiles del grupo dominante a fin de influir sobre este, y una apariencia de desamparo e ignorancia bajo la que se oculta un fraudulento deseo de dominio ${ }^{3}$.

En las mujeres hay diferentes variables de estigmatización:

- Cada mujer es tratada como "una mujer", sus cualidades y sus logros resultan secundarios.

- Las mujeres son vistas como "todas iguales" y por lo tanto sustituibles unas por otras.

- Se les impone un estatuto de secundonas, a las que se considera pasivas, y por lo tanto susceptibles de ser tratadas como un objeto.

- La subordinación significa, además, que pueden sufrir fácilmente: acoso, violencia, discriminación.

- Pueden ser ignoradas, o descartadas, o trivializadas, tratadas como niñas o como no personas.

- Su status social es visto como logrado vicariamente a través de los hombres ${ }^{4}$.

\section{Diferencias de Género}

Cuando se habla de género se diferencia del término sexo. En general los sociólogos utilizan el término sexo para referirse a las diferencias anatómicas y fisiológicas que definen las diferencias del cuerpo del hombre y de la mujer. Por el contrario, el género afecta a las diferencias culturales, psicológicas y sociales que existen entre el hombre y la mujer. Algunos autores sostienen que hay ciertos aspectos biológicos (hormonas, tamaño del cerebro, genética) que son responsables de la diferencia de comportamiento entre mujeres y hombres. Por ello en casi todas las culturas los hombres se dedican a la caza y a la guerra. Pero las mujeres pasan mucho tiempo cuidando a los hijos y no les sería fácil participar en la guerra o en la caza. 
Aunque existe esta hipótesis de que los factores biológicos influyan en el comportamiento diferenciado de uno y otro sexo, un siglo de investigaciones sobre el origen fisiológico no ha podido demostrarla.

Otra vía para comprender las diferencias en función del género es el estudio de la socialización del género, el aprendizaje de unos determinados roles.

Pero ¿cómo se mantiene esta reproducción social? Pues con el control social en el que encontramos siempre relaciones de poder. El control social se inicia en la familia, escuela, ámbito laboral. Tradicionalmente la sociedad patriarcal ha venido penalizando con mecanismos diversos de control social la trasgresión de la división sexual del trabajo Estos comportamientos, ideas, creencias respecto de los hombres y mujeres llegará a ser tan potente que aunque alguna evidencia se oponga a dichas creencias algunos individuos continúan manteniéndolas a lo largo de su vida.

Según Giddens no existe sociedad conocida en la que las mujeres sean más poderosas que los hombres. En la gran mayoría de sociedades las mujeres se han venido dedicando prioritariamente al cuidado de los hijos y del hogar mientras que los hombres se han ocupado de las actividades políticas y militares ${ }^{5}$. Pero la importancia de la mujer aún fue debilitada mucho más con la Revolución Industrial. En el antiguo orden, las mujeres se habían ganado la supervivencia participando en el trabajo compartido de la familia. La mujer tejía, curaba, pues conocía las hierbas capaces de sanar, plantaba la huerta, alimentaba el ganado, hacía mantequilla y queso, hacía conservas... Era subordinada, pero no dependiente y desamparada. Con la nueva economía de mercado, la antigua unidad entre trabajo, hogar y reproducción se rompe y quedan completamente divididas las dos vidas "pública y privada". Todo lo anterior fue derrocado y las vidas de las mujeres entraron en confusión. Fuera de casa la mujer no podía ganarse la vida, y para una mujer no había vida. Los médicos, psicólogos, expertos en nutrición... se convirtieron en los nuevos expertos y las mujeres se dejaron aconsejar por ellos. Nunca fue una relación entre iguales, sino nada más que la ideología de una sociedad machista disfrazada de verdad objetiva ${ }^{6}$.

Todo ello junto con los avances tecnológicos llevaba consigo un conjunto de cambios que no solo la mujer debía realizar sino también el hombre. Pero con la carga cultural llevada a cuestas durante años era difícil de producirse sin grandes conflictos, y por desgracia, las víctimas fueron la mayoría mujeres. Las mujeres intentaron afrontar estas desigualdades a medida que adquirían conciencia de ello, las que lo hacían pasaban a formar parte de una lucha activa, y como comenta B. Ehrenreich otras, igual de cultas y formadas, caían en depresión y enfermedad.

\section{Feminismo}

Los movimientos de protesta van apareciendo a la vez que la mujer va adquiriendo reconocimiento de su situación, pero es a partir de 1960 cuando la teoría feminista toma conciencia para la sociología. Aunque todas las teorías feministas toman conciencia sobre la desigualdad de la mujer, hay algunas diferencias entre ellas.

El movimiento que articuló la lucha de las mujeres fue el feminismo. Hay acusados contrastes entre las diferentes teorías feministas relacionadas con la desigualdad de género. Aunque a todas les preocupa la desigualdad de la mujer en la sociedad, las explicaciones que dan son muy diferentes. Los tres enfoques feministas principales son el liberal, el radical y el feminismo negro: 
Feminismo Liberal: Mediante explicaciones marxistas de la posición de la mujer, las mujeres son explotadas y explotan en parte sobre la base del género y en base sobre la posición de clase.

Feminismo radical: Los hombres son los responsables de la explotación de la mujer y se benefician de ella. La raíz es la opresión patriarcal que hace que los hombres usen su fuerza bruta para someter a otros. Para ellas, como el patriarcado es un fenómeno sistémico, la igualdad entre los géneros solo podrá obtenerse derrocando el orden patriarcal.

Feminismo negro: Señalan que las principales escuelas del pensamiento feminista no tienen en cuenta divisiones de tipo étnico que existen entre las mujeres, porque dichas corrientes se centran en los dilemas de la mujer blanca, especialmente de clase media, que vive en sociedades industrializadas. Este tipo de feminismo se centra en los problemas concretos de las mujeres negras. Tienen en cuenta la influencia que ha tenido en ellas el legado de la esclavitud, la segregación y el movimiento de derechos civiles sobre las desigualdades de género que afectan a las comunidades negras ${ }^{2}$.

\section{Trabajo y Mujer}

Dentro del mercado de trabajo podemos observar unas áreas feminizadas mientras otras han sido vetadas a la mujer. Al igual en las familias, aunque muchas veces inconscientemente han intentado utilizar la teoría del capital humano, es decir, invertir más en los hombres pues serían estos los que tenían que mantener una familia, mientras que la mujer siempre tenía el recurso del matrimonio. La supuesta eficacia de las mujeres en el trabajo doméstico deriva, no obstante, únicamente de su capacidad biológica para criar y amamantar. Es la valoración social que se da a las tareas masculinas, lo que excluye a las mujeres del trabajo cualificado y lo que lleva a la conclusión de que los hombres adquieren cualificaciones y las mujeres poseen cualidades.

Las teorías de los sistemas duales afirman que la dominación en la esfera privada tiene lugar por medio del patriarcado, mientras que la segunda opresión de clase pertenece a la esfera pública y opera por medio del capitalismo. Aunque pueden reforzarse mutuamente a la vez se encuentran en tensión, pues el primero quiere conservar a las mujeres en casa, mientras que el segundo las quiere trabajando fuera de casa ${ }^{7}$.

Pese a todo el esfuerzo realizado por las luchas feministas, el reparto de responsabilidades en el hogar dista todavía de ser igualitario. Por otra parte en lo que hace referencia a la incorporación de la mujer en algunos lugares de trabajo y de poder su presencia todavía es mínima. El desarrollo armónico sería aquel que permitiera una participación igualitaria tanto en el marco laboral como en el hogar.

Pero si como ocurre normalmente la mujer tiende a dedicar una proporción menor de su vida activa al mercado de trabajo, y más capital humano doméstico no podrá cambiar la situación actual. Para ello necesitamos que haya un cambio de las actitudes de los hombres, difícil para aquellos que han estado socializados con una división estricta de roles, pero no imposible. Representa una pérdida de poder y 
estatus, pues aquellos puestos antes ocupados por ellos serán reemplazados poco a poco.

La gente que opta por el divorcio es cada vez mayor, sobre todo cuando las mujeres tienen un trabajo remunerado que les permita una independencia económica. También cabe destacar que las tareas domésticas siguen siendo realizadas en su mayoría por mujeres, y si se ha reducido el tiempo medio de estas ha sido por al aumento de electrodomésticos que le facilitaban las tareas y le permitían ahorrar tiempo. Algunos jóvenes todavía desprecian las tareas domésticas. Los padres no les han inculcado esta necesidad pues para ellos era prioritario por coste/beneficio inculcarles otras más productivas. Sería importante la difusión de nuevos valores y formación de hombres adultos y jóvenes la preparación en cursos de gestión, administración doméstica, puericultura.

Sería importante empezar esta educación precozmente los padres junto con la escuela iniciando campañas de educación, diálogo y debate, siguiendo en el Instituto y en la universidad. La formación abre la trampa: posibilita una carrera profesional, y por tanto la evasión de la estrechez de la existencia como ama de casa, quita la legitimación a las oportunidades de conseguir trabajo y libera la búsqueda del matrimonio como medio de subsistencia. Pero todo ello no ha eliminado las desigualdades, aunque se ven con más claridad ${ }^{8}$.

Pero la cuestión de las capacidades preocupa a los hombres. El aparente ascenso de las mujeres, sus mejores rendimientos académicos, lleva a interrogarse a los hombres sobre sus propias capacidades.

\section{Violencia y Mujer}

En los países industrializados la mayoría de mujeres se considera igual a los hombres, con sus mismos derechos y además el del control de sus cuerpos y sus vidas. Es la revolución más importante porque llega a la raíz de la sociedad. Pero al igualarnos respecto a los hombres la violencia interpersonal y el maltrato psicológico aumenta, debido a la ira de los hombres, individual y colectiva por su pérdida de poder.

Los mandatos culturales y a menudo también los legales sobre los derechos y privilegios del marido han legitimado históricamente un poder y dominación del marido sobre la mujer, promoviendo su dependencia económica de él y garantizando el uso de la violencia y de las amenazas para controlarla. Los hombres en general aceptan más que las mujeres la violencia matrimonial, y los hombres con roles más tradicionales son los que más la aceptan. Una característica común a los agresores es la necesidad de control y dominio combinada con una percepción que indica que la autonomía femenina implica una pérdida del control del hombre.

La educación sexual en nuestra sociedad más que tener un origen natural y biológico, lo tiene social, lo cual refleja la posición subordinada de mujeres y adolescentes en nuestra sociedad ${ }^{9}$.

Dentro de la violencia contra las mujeres podemos incluir el tráfico de mujeres, pues la mayoría de veces son engañadas y no saben que acabarán trabajando en un burdel. El tráfico de mujeres y la prostitución se reconocen como una violación de los 
derechos humanos, para la Conferencia de las Naciones Unidas sobre Derechos Humanos (1993) ${ }^{10 .}$

El concepto de tráfico de mujeres se amplía a otras formas de explotación como trabajos forzados y prácticas esclavistas como resultado de la situación de pobreza legal y social de las mujeres. Es importante asegurarles sus derechos legales y sociales como mujeres, como inmigrantes, como trabajadoras y como amas de casa. Si esos derechos no son reconocidos, las prácticas de esclavitud y el trabajo forzado continuarán existiendo.

El turismo sexual está relacionado con los altos índices de desempleo y con la precariedad laboral. Algunas familias desesperadas, obligan a sus hijas a prostituirse. Otras mujeres desean abandonar el ambiente tradicional de su lugar de origen y se ven forzadas a realizarlo pues no encuentran oportunidades en la gran ciudad. Con frecuencia la prostitución está relacionada con la violencia, delincuencia, tráfico de drogas, la explotación y la violación de los derechos humanos.

La prostitución expresa las desigualdades de poder entre hombres y mujeres, pero también hay que tener en cuenta otros factores, la prostitución proporciona un medio de conseguir satisfacción sexual a quienes por problemas físicos o por la existencia de códigos morales restrictivos no consiguen encontrar otras parejas.

Durante mucho tiempo se creía que la mujer no tenía orgasmo, este solo podía ser atribuido al hombre pues el papel pasivo y secundario era de la mujer. Incluso durante un tiempo persistió el modelo de sexo único. En definitiva la heterosexualidad institucionaliza el dominio sexual del varón y la sumisión sexual de la mujer ${ }^{11 .}$

\section{Globalización}

El capitalismo en la actualidad va de la mano de una de las revoluciones más importantes en nuestra civilización que es la científico-tecnológica. Acompañada de la automatización de ciertos procesos fabriles, robotización, utilización de Internet, ha provocado y lo está haciendo todavía, numerosos cambios sociales que están socavando las bases de la familia patriarcal y están haciendo una sociedad todavía más dualizada y con más desigualdades. Es la sociedad "en red" que ha descrito Castells. La mayor parte de la sociedad de Europa Occidental sigue disfrutando de los niveles de vida más altos del mundo. Pero claro, nos referimos a medias y no a otros segmentos de población que igualmente en los países desarrollados vive en situación de miseria o de pobreza. Un caso extremo de desigualdad es el de desnutrición que afecta a 815 millones de personas como recuerda la FAO.

El clima de deterioro social se ve afectado especialmente por los procesos de precariedad laboral, de esta manera, los jóvenes, las mujeres, los inmigrantes y las personas con cualificaciones más bajas se están viendo sometidos a unas condiciones laborales e ingresos cada vez más deteriorados. Mientras otro segmento de población está entrando en una dinámica de consumos cada vez más ostentosos y en un consumo casi compulsivo. Como vemos las mujeres vuelven a encontrarse dentro de este colectivo de dualización en mayor parte que otros segmentos de población.

El capitalismo seguirá buscando ganancias en un mundo donde los obstáculos para su movilidad van desapareciendo. Se internacionalizan los procesos de trabajo para 
aumentar las ganancias, existen menos regulaciones fiscales y productivas y procesos que escapan del control estatal. Estamos pues en una fase que podríamos denominar "capitalismo global" 12.

Dentro de esta búsqueda de beneficios un modo de aumentarlos ha sido el aumento de contratación de las mujeres como mano de obra barata, muchas de ellas han abandonado el campo y se han concentrado en zonas francas que es donde se han instalado las multinacionales (Irlanda, isla de Mauricio, Bangla Desh.). Aunque la asalarización de estas mujeres trae consigo efectos positivos como son la emancipación de las relaciones patriarcales en el hogar, lleva consigo también efectos negativos que son los efectos de producción opresiva y explotadora. La emigración trae consigo el aumento de prostitución y el empleo de las mujeres en aquellas tareas típicamente femeninas que son las de cuidado y del mantenimiento del hogar que tan desvalorizado ha estado siempre, así como los servicios sexuales.

\section{Profesiones}

En el Sistema Sanitario también se reproducen los estereotipos sociales y en relación al poder de las enfermeras el paralelismo es evidente y sus consecuencias han provocado grandes obstáculos ${ }^{13}$.

Mientras la enfermería española no consiga insertarse en los niveles superiores del sistema educativo, trascendiendo el grado medio, aunque éste sea impartido por y en la Universidad, no logrará una equiparación efectiva con la medicina, ni la enfermera con el médico, perpetuándose las relaciones de dominación ${ }^{14}$.

En la sociedad actual nos acercamos progresivamente a la igualdad tan esperada, tanto en nivel familiar como en el profesional. No obstante mujeres y hombres debemos adaptarnos al entorno tan cambiante con el que interrelacionamos, el cual requiere una constante preparación y formación. Todos los cambios generan estrés para ambos géneros, pero más para las mujeres que han tenido que desarrollar la función de bisagra o como denomino en algún otro trabajo "mujeres transicionales" que muchas veces han pagado con su cuerpo o con su mente el liderazgo que se les ha encomendado.

\section{BIBLIOGRAFIA}

1. Castells M. El fin del patriarcado: movimientos sociales, familia y sexualidad en la era de la información. La era de la información: economía, sociedad y cultura. Madrid: Alianza Editorial, 1997. pp.159.

2. Giddens A. Género y sexualidad, Sociología. Madrid: Alianza Universidad, 2002 (4⿳亠丷厂 ed). pp: 156-158.

3. Vázquez K. Las categorías de sexo, género y sexualidad: la construcción de las mujeres como sujetos políticos. En: Arantza Campos y Lourdes Méndez, Teoría feminista: identidad, género y política. San Sebastián: Servicio editorial de la Universidad del País Vasco, 1993.

4. Osborne R. ¿Son las mujeres una minoría? Isegoría. 1996, octubre. 14:79-93.

5. Brullet i Tenas C. Roles e identidades de género: una construcción social. Sociología de las mujeres españolas. Madrid: Ed.Complutense,1996, pp: 280-85.

6. Ehrenreich, B. y English, D. Propio bien (150 años de consejos de expertos a las mujeres). Madrid: Taurus, 1990, Cap 1. introd., pp: 14-23. 
7. Ferreira, V. Mujer y trabajo. Mujer y Trabajo. La división sexual del trabajo en el análisis sociológico: de natural a socialmente construida. En: María Antonia García de León, Félix Ortega y Maria Luisa García de Cortazar. Sociología de las mujeres españolas. Madrid: E. Complutense, 1996, pp: 97-100.

8. Beck, U. y Beck-Gernsheim. El normal caos del amor. Las nuevas formas de relación amorosa, Barcelona: Paidós, 2001.

9. Lorente Acosta M, Lorente Acosta JA. Agresión a la mujer: maltrato, violación y acoso. Madrid: Ed Comares, 1998. pp: 45-48.

10.Wijers, M. Política de la Unión Europea sobre el tráfico de mujeres. Madrid: Narcea, 2001, pp: 245-266.

11. Laqueur T. La construcción del sexo. Cuerpo y género desde los griegos hasta Freud. Madrid: Cátedra, 1990.

12. Benería L. La globalización de la economía y el trabajo de las mujeres. Revista de economía y sociología del trabajo. 1991, 113-114:23-25.

13. Alberdi R. Mujeres Sanitarias: una historia de género y debate. Bioética y Debate. 1999; 4(16):11-15.

14. Martín C. Enfermería, presente y futuro. Madrid: Colegio Oficial de ATS. 1978. 